\title{
Nutrients or nursing? Understanding how breast milk feeding affects child cognition
}

\author{
Wei Wei Pang ${ }^{1} \cdot$ Pei Ting Tan ${ }^{1}$ - Shirong Cai ${ }^{1,2}$ - Doris Fok ${ }^{1} \cdot$ Mei Chien Chua ${ }^{3}$. Sock Bee Lim ${ }^{4} \cdot$ Lynette P. Shek $^{2,5,6}$. \\ Shiao-Yng Chan ${ }^{1,2} \cdot$ Kok Hian Tan $^{7,8} \cdot$ Fabian Yap $^{9} \cdot$ Peter D. Gluckman 2,10 $\cdot$ Keith M. Godfrey ${ }^{11,12}$. \\ Michael J. Meaney ${ }^{2,13,14} \cdot$ Birit F. P. Broekman ${ }^{15} \cdot$ Michael S. Kramer $^{1,16,17} \cdot$ Yap-Seng Chong ${ }^{1,2} \cdot$ Anne Rifkin-Graboi ${ }^{2,18}$
}

Received: 2 October 2018 / Accepted: 9 February 2019 / Published online: 26 February 2019

(c) The Author(s) 2019

\begin{abstract}
Purpose To explore the associations between type of milk feeding (the "nutrients") and mode of breast milk feeding (the "nursing") with child cognition.

Methods Healthy children from the GUSTO (Growing Up in Singapore Toward healthy Outcomes) cohort participated in repeated neurodevelopmental assessments between 6 and 54 months. For "nutrients", we compared children exclusively bottle-fed according to type of milk received: formula only $(n=296)$ vs some/all breast milk $(n=73)$. For "nursing", we included only children who were fully fed breast milk, comparing those fed directly at the breast $(n=59)$ vs those fed partially/completely by bottle $(n=63)$.

Results Compared to infants fed formula only, those who were bottle-fed breast milk demonstrated significantly better cognitive performance on both the Bayley Scales of Infant and Toddler Development (Third Edition) at 2 years [adjusted mean difference $(95 \%$ CI $1.36(0.32,2.40)]$, and on the Kaufman Brief Intelligence Test (Second Edition) at 4.5 years [7.59 $(1.20,13.99)]$. Children bottle-fed breast milk also demonstrated better gross motor skills at 2 years than those fed formula $[1.60(0.09,3.10)]$. Among infants fully fed breast milk, those fed directly at the breast scored higher on several memory tasks compared to children bottle-fed breast milk, including the deferred imitation task at 6 months $[0.67(0.02,1.32)]$ and relational binding tasks at $6[0.41(0.07,0.74)], 41[0.67(0.04,1.29)]$ and $54[0.12(0.01,0.22)]$ months.

Conclusions Our findings suggest that nutrients in breast milk may improve general child cognition, while nursing infants directly at the breast may influence memory.
\end{abstract}

Keywords Breastfeeding $\cdot$ Breast milk expression $\cdot$ Child cognition $\cdot$ Memory

\section{Introduction}

Though non-unanimous, numerous observational studies, meta-analyses, and randomized trial suggest breastfeeding improves child cognition [1-6]. Breastfeeding's benefits appear greatest in studies of young children [6]. Several

Wei Wei Pang and Pei Ting Tan contributed equally.

Electronic supplementary material The online version of this article (https://doi.org/10.1007/s00394-019-01929-2) contains supplementary material, which is available to authorized users.

Anne Rifkin-Graboi

anne.rifkin@nie.edu.sg

Extended author information available on the last page of the article hypotheses may explain the association between breastfeeding and cognitive ability.

First, the benefits may be due to the nutritional contents of breast milk, like long-chain fatty acids such as docosahexaenoic acid (DHA) and arachidonic acid (AA), and their influence on brain development. DHA and AA together comprise approximately $20 \%$ of the brain's fatty acid content and are involved in several aspects of early neurodevelopment, including modulation of cell growth and membrane lipid biosynthesis and myelination [7, 8]. Beyond fatty acids, breast milk also contains sialic acid, a key building block of brain ganglioside [9, 10], and other important nutrients for myelin synthesis, such as zinc, choline, and vitamin $B_{12}$ [11]. Indeed, breastfeeding is linked to a faster rate of white matter development in brain regions associated with highorder cognition [12]. 
Second, breastfeeding might exert effects through the physical and/or emotional contact between mother and infant during breastfeeding [13, 14]. For example, greater maternal brain activation in response to breastfeeding has been associated with improved maternal sensitivity [15], which in turn is positively associated with infant language development [16]. Moreover, it is reasonable to think that direct breastfeeding associates with increased mother-child physical contact, and perhaps, skin-to-skin contact, which along with other forms of variation in exposure to maternal touch predict neurodevelopment [17].

Previous published studies on breastfeeding and child cognition have analyzed breastfeeding in terms of its duration and exclusivity. To our knowledge, these studies have not assessed whether associations with child cognition resulted from breast milk nutrients, the physical/emotional contact during breastfeeding, or a combination of both. Previous studies have not examined the relationship between breastfeeding mode-feeding directly at the breast vs feeding expressed breast milk (usually by bottle)—and child cognition, despite the increasing worldwide trend toward breast milk expression [18-20]. One randomized trial demonstrated a large benefit in cognition when preterm infants were tube fed breast milk vs infant formula, suggesting a positive effect of breast milk nutrients, but none of the infants received direct breastfeeding during hospitalization [21].

We previously reported significant associations between breastfeeding and child cognition among healthy, term infants in the first 2 years of life in the 'Growing Up in Singapore Toward healthy Outcomes' (GUSTO) study, comprised of multi-ethnic Asian Singaporeans [4]. We have also shown that breast milk expression is common, with a substantial fraction of GUSTO mothers feeding their infants expressed breast milk only instead of feeding directly at the breast [22]. Here, we use data from the same prospective cohort to explore the associations between mode of breast milk feeding (the "nursing") and type of milk fed (the "nutrients", i.e., breast milk vs formula) and child cognition, with a broad range of cognitive outcomes now extended to 4.5 years, and hypothesize that both "nursing" at the breast and the "nutrients" in breast milk feeding influence child cognitive ability.

\section{Methods}

\section{Study design and population}

In 2009 and 2010, women in their first trimester of pregnancy who were 18-46 years of age and of homogeneous (both parents) Chinese, Malay or Indian ethnicity were recruited from KK Women's and Children's Hospital (KKH) and National University Hospital (NUH) in Singapore into the GUSTO birth cohort study [23]. All children were offered a neurodevelopmental assessment at 48 months. Owing to limited availability of the evaluators, however, only a subset of children participated in the assessments conducted at $6,18,24,41$ and 54 months. The study was approved by the National Healthcare Group Domain Specific Review Board (NHG DSRB) and the Sing Health Centralised Institutional Review Board (CIRB). All participating mothers provided written informed consent.

Of 1247 mother-child dyads recruited, we excluded dyads from analyses if offspring were: not singletons; born preterm ( $<37$ weeks gestation); from pregnancies with complications (e.g., pre-eclampsia, gestational diabetes); with birth weight $<2500 \mathrm{~g}$ or $>4000 \mathrm{~g}$; or had a last recorded Apgar score of $<9$ at 5 or 10 min post-delivery (Fig. 1a, b).

For "nursing" analyses, comparing different modes of feeding breast milk, only children who were fully fed breast milk at 3 months postpartum were included $(n=122)$ (Fig. 1a). As detailed previously [22], fully breastfed included infants who were either exclusively breastfed (i.e., only received breast milk, including expressed breast milk) or those who were predominantly breastfed (i.e., received breast milk and may have received some non-milk liquids such as water and water-based drinks [including oral rehydration solution, fruit juices], or syrups and drops consisting of vitamins, minerals or medications). Very few children (2.5-3\%) were predominantly breastfed in our cohort [22], with most of these predominantly breastfed infants receiving water, rather than other non-milk liquids. For "nutrient" analyses, comparing the consumption of breast milk vs formula, we included only children who were exclusively bottle-fed at 3 months postpartum $(n=369)$ (Fig. 1b).

The number of children with available neurocognitive data at each time point is indicated in Fig. 1. As some children had unusable data owing to fatigue, poor cooperation or fussiness, as well as technical errors (e.g., computer or video malfunction) particularly at 6 and 18 months, the number of children with usable data for each task differed.

\section{Data collection}

Participants' ethnic backgrounds, recruitment age and highest educational attainment were obtained from mothers at $<14$ weeks gestation by trained research coordinators. Pregnancy complications (pre-eclampsia and gestational diabetes) and delivery details (gestational age, infant sex, Apgar scores, and birth weight) were extracted from medical records. Infants were classified into birth weight percentiles as described by Mikolajczyk et al. [24]. Mothers completed the State-Trait Anxiety Inventory (STAI) at 26-28 weeks' gestation, as detailed previously in the GUSTO cohort [25].

Infant feeding type (exclusive breastfeeding, predominant breastfeeding, partial breastfeeding or formula only) and 
Fig. 1 Flowchart of participants for analyses examining neurodevelopmental outcomes among different a types of nursing and b milk nutrients

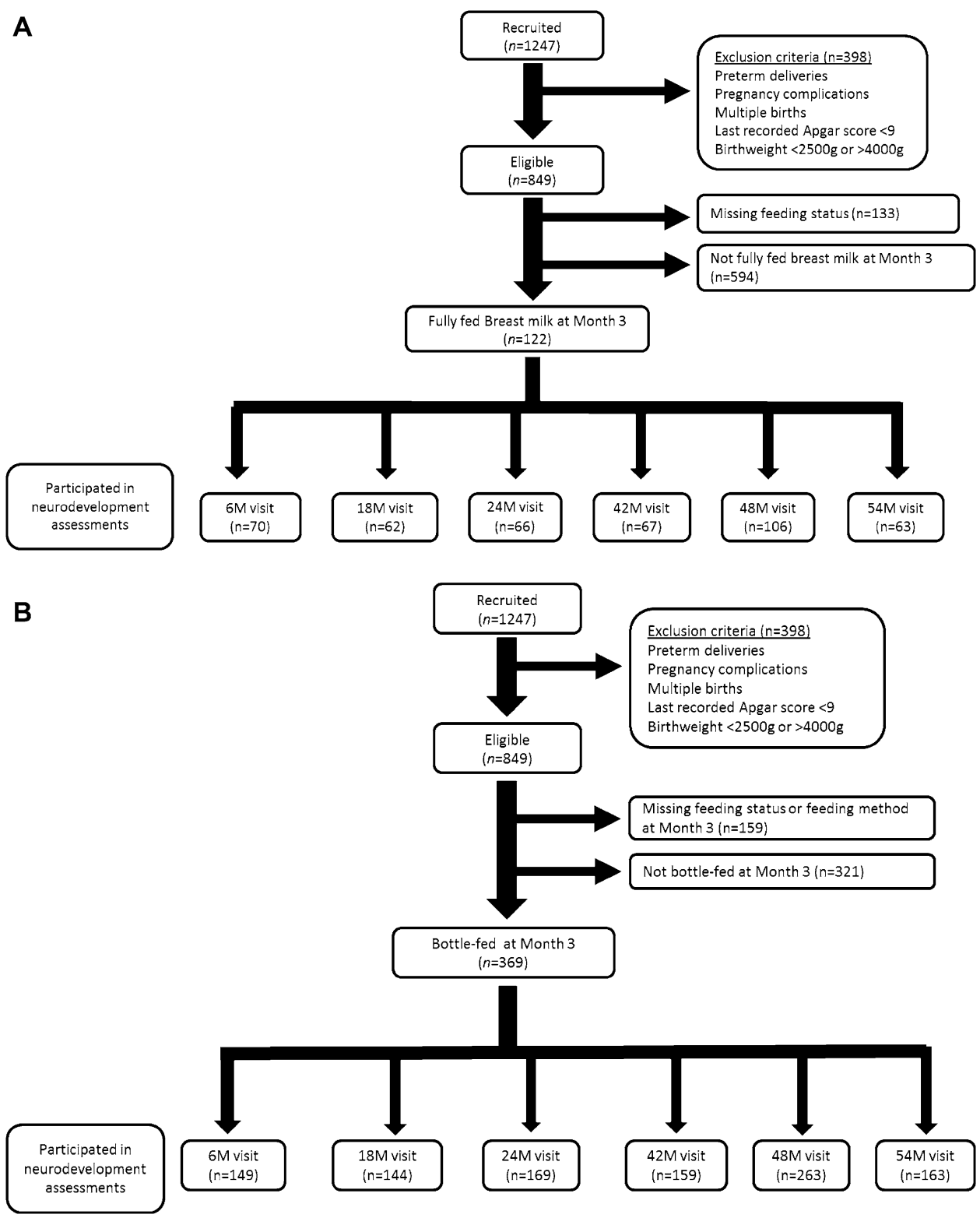

data pertaining to the age of breastfeeding cessation were ascertained at week 3, month 3 and every 3-month intervals thereafter until 12 months using interviewer-administered questionnaires. Any breast milk feeding refers to an infant receiving breast milk (either directly at the breast or fed expressed breast milk), with or without non-human milk and/or solids. At 3 months, breastfeeding mothers were asked how their infants were fed breast milk (at the breast, bottle only, and breast + bottle) [26]. Bottle only includes infants who received only breast milk expressed from the breast (either manually or via a pump) by bottle, cup or spoon (very few were fed by cup or spoon). Breast + bottle refers to infants fed directly at the breast but who also received some expressed breast milk by bottle (or cup or spoon).
Our primary outcome was child cognition assessed from 6 to 54 months. Neurocognitive assessments conducted at the different time points included paper and pencil/computerized tasks requiring motor and/or verbal responses, behavioral observation and eye tracking (Table 1). These assessments were conducted by personnel trained by GUSTO cohort investigators; for standardized tests like the Bayley Scales of Infant and Toddler Development, 3rd edition (BSID-III) and the Kaufman Brief Intelligence Test, 2nd edition (KBIT-2), personnel were trained by a psychologist/psychiatrist. With the exception of BSID-III and School Readiness Test which were conducted at participant's home at 24 months and 48 months, respectively, all other neurocognitive assessments were performed at the clinic. The full details of the cognitive 
Table 1 Summary of neurocognitive assessments in 6-54-month-old children

\begin{tabular}{|c|c|c|c|c|c|c|}
\hline \multirow[t]{2}{*}{ Type of tasks } & \multicolumn{6}{|l|}{ Time points } \\
\hline & 6 months & 18 months & 24 months & 41 months & 48 months & 54 months \\
\hline Memory & $\begin{array}{l}\text { Habituation } \\
\text { Deferred imitation } \\
\text { Relational binding }\end{array}$ & Deferred imitation & Deferred imitation & $\begin{array}{l}\text { Deferred imitation } \\
\text { Relational Binding }\end{array}$ & & Relational Binding \\
\hline $\begin{array}{l}\text { Executive func- } \\
\text { tioning and self- } \\
\text { regulation }\end{array}$ & & & & $\begin{array}{l}\text { Dimensional Card } \\
\text { Sorting Task } \\
\text { Snack \& sticker } \\
\text { delay }\end{array}$ & & $\begin{array}{l}\text { Dimensional Card } \\
\text { Sorting Task }\end{array}$ \\
\hline $\begin{array}{l}\text { Attention/ } \\
\text { pre-attention } \\
\text { and working } \\
\text { Memory }\end{array}$ & Visual expectation & Visual expectation & & & & $\begin{array}{l}\text { CANTAB - spatial } \\
\text { working memory }\end{array}$ \\
\hline $\begin{array}{l}\text { Social-emotional } \\
\text { development }\end{array}$ & & & & & & Novel word learning \\
\hline Testing batteries & & & $\begin{array}{l}\text { Bayley Scales of } \\
\text { Infant Develop- } \\
\text { ment III }\end{array}$ & & $\begin{array}{l}\text { School readiness } \\
\text { test }\end{array}$ & $\begin{array}{l}\text { Kaufman Brief } \\
\text { Intelligence test - } 2\end{array}$ \\
\hline
\end{tabular}

Assessment details and references are shown in the Supplementary Methods (Online Resource 1)

test methodologies are provided in the Supplementary Methods (Online Resource 1).

\section{Statistical analyses}

We conducted two separate analyses. In our "nursing" analyses, we analyzed breast milk feeding mode by including only children who were fully fed breast milk at 3 months. In this analysis, we compared those who were fed only directly at the breast; those fed directly at the breast who also received expressed breast milk (either manually or via a pump) by bottle, cup or spoon; and those who received only expressed breast milk. Since very few $(n=11)$ infants received expressed breast milk only, they were combined with the middle (direct+expressed) group (Supplemental Table 1, Online Resource 1).

In our "nutrient" analyses, we compared groups of children who were exclusively bottle-fed but who differed in the type of milk received: breast milk, formula, or a combination of both. Infants who were fed at the breast, either exclusively or partially, were excluded from the second analysis. Again, because very few $(n=11)$ infants were bottle-fed breast milk only, they were added to the combination group (Supplemental Table 1, Online Resource 1).

Cohort participants are described using proportions or means $\pm \mathrm{SD}$, with crude (unadjusted) comparisons of the types of nursing and milk nutrients based on Chi square tests or $t$ tests. Adjusted associations of the types of nursing and milk nutrients with neurocognitive outcomes were examined using multivariable linear regression or logistic regression for continuous or dichotomous outcomes, respectively.
The choice of covariates included in multivariable models was based on our previous studies [27, 28]: ethnicity (Chinese, Malay, or Indian), maternal education (tertiary and non-tertiary), child's sex, birth weight category [small for gestational age (SGA), appropriate for gestational age (AGA), and large for gestational age (LGA)], and antenatal maternal STAI-state scores. Participants (0-6\%) with missing covariates were excluded from the statistical analyses. Sensitivity analyses using multiple imputation were also conducted; the results were similar and are, therefore, not presented. All statistical analyses were performed using SPSS version 24.0 (IBM Corp., Armonk, NY, USA).

\section{Results}

\section{Participant characteristics}

Among mothers who were feeding breast milk fully at 3 months, similar proportions fed their infants directly at the breast (48.4\%) or partially/completely fed their infants breast milk by bottle (51.6\%) (Table $2 \mathrm{a}$ ). Girls and children of mothers without tertiary education, tended to be fed directly at the breast, rather than bottle-fed breast milk. Breast milk feeding duration was similar between the two groups. Among all mothers who bottle-fed their infants at 3 months, the majority of mothers fed their infants formula exclusively $(80.2 \%)$, with $19.8 \%$ mothers feeding their infants some or all expressed breast milk (Table 2b). Mothers of Malay ethnicity, of younger age, without tertiary education or who were more anxious during pregnancy were more likely to bottle-feed their infants formula only. Not 
Table 2 Maternal and infant characteristics by (a) type of nursing and (b) milk nutrients at 3 months postpartum

\begin{tabular}{|c|c|c|c|c|c|c|c|c|}
\hline \multirow[t]{2}{*}{ Characteristics } & \multicolumn{4}{|c|}{ (a) Nursing (breast milk only) } & \multicolumn{4}{|c|}{ (b) Nutrients (fed by bottle) } \\
\hline & $\begin{array}{l}\text { All par- } \\
\text { ticipants } \\
(n=122)\end{array}$ & $\begin{array}{l}\text { At breast } \\
(n=59 \\
48.4 \%)\end{array}$ & $\begin{array}{l}\text { Breast }+ \text { bottle or } \\
\text { bottle only }(n=63 \text {, } \\
51.6 \%)\end{array}$ & $p$ & $\begin{array}{l}\text { All par- } \\
\text { ticipants } \\
(n=369)\end{array}$ & $\begin{array}{l}\text { Formula only } \\
(n=296, \\
80.2 \%)\end{array}$ & $\begin{array}{l}\text { Breast milk }+ \text { for- } \\
\text { mula or breast milk } \\
\text { only }(n=73,19.8 \%)\end{array}$ & $p$ \\
\hline Ethnicity & & & & 0.057 & & & & $<0.001$ \\
\hline Chinese & 85 & $36(42.4)$ & $49(57.6)$ & & 205 & $140(68.3)$ & $65(31.7)$ & \\
\hline Malay & 16 & $8(50.0)$ & $8(50.0)$ & & 108 & $106(98.1)$ & $2(1.9)$ & \\
\hline Indian & 21 & $15(71.4)$ & $6(28.6)$ & & 56 & $50(89.3)$ & $6(10.7)$ & \\
\hline $\begin{array}{l}\text { Maternal age (year), } \\
\text { mean } \pm \text { SD }\end{array}$ & $30.9 \pm 4.4$ & $31.0 \pm 4.6$ & $30.8 \pm 4.2$ & 0.814 & $29.4 \pm 5.3$ & $28.9 \pm 5.4$ & $31.6 \pm 4.4$ & $<0.001$ \\
\hline Maternal education ${ }^{\mathrm{a}}$ & & & & 0.001 & & & & $<0.001$ \\
\hline Non-tertiary & 39 & $27(69.2)$ & $12(30.8)$ & & 281 & $256(91.1)$ & $25(8.9)$ & \\
\hline Tertiary & 79 & $30(38.0)$ & $49(62.0)$ & & 83 & $35(42.2)$ & $48(57.8)$ & \\
\hline Child's sex & & & & 0.019 & & & & 0.600 \\
\hline Male & 63 & $24(38.1)$ & $39(61.9)$ & & 187 & $148(79.1)$ & $39(20.9)$ & \\
\hline Female & 59 & $35(59.3)$ & $24(40.7)$ & & 182 & $148(81.3)$ & $34(18.7)$ & \\
\hline $\begin{array}{l}\text { Child's birth weight } \\
\text { category }\end{array}$ & & & & 0.462 & & & & 0.786 \\
\hline SGA $(<10 \%)$ & 10 & $4(40.0)$ & $6(60.0)$ & & 44 & $36(81.8)$ & $8(18.2)$ & \\
\hline AGA (10-90\%) & 94 & $44(46.8)$ & $50(53.2)$ & & 278 & 224 (80.6) & $54(19.4)$ & \\
\hline LGA (>90\%) & 18 & $11(61.1)$ & 7 (38.9) & & 47 & $36(76.6)$ & $11(23.4)$ & \\
\hline $\begin{array}{l}\text { STAI-state } \\
\text { anxiety at } 26 \\
\text { week pregnancy, }{ }^{\text {a }} \\
\text { mean } \pm \text { SD }\end{array}$ & $31.0 \pm 8.7$ & $31.6 \pm 8.8$ & $30.5 \pm 8.5$ & 0.504 & $35.4 \pm 10.0$ & $36.2 \pm 10.1$ & $31.9 \pm 8.7$ & 0.001 \\
\hline $\begin{array}{l}\text { Duration of any } \\
\text { breast milk feed- } \\
\text { ing (month) } \\
\text { mean } \pm \text { SD }\end{array}$ & $12.5 \pm 3.3$ & $12.7 \pm 3.1$ & $12.2 \pm 3.5$ & 0.405 & $2.0 \pm 2.9$ & $1.0 \pm 0.8$ & $7.3 \pm 4.0$ & $<0.001$ \\
\hline
\end{tabular}

Data presented are $n(\%)$ unless otherwise stated

$A G A$ appropriate for gestational age, $L G A$ large for gestational age, $S G A$ small for gestational age, STAI State-Trait Anxiety Inventory

${ }^{a}$ Number of participants with missing data: maternal education, (a) $n=4$, and (b) $n=5$; STAI-state Anxiety at 26 week pregnancy, (a) $n=4$, and (b) $n=2$; duration of any breast milk feeding, (a) $n=18$, and (b) $n=43$

surprisingly, the duration of breast milk feeding was significantly longer among mothers who fed their infants some or all expressed breast milk when compared to those who fed their infants formula only at 3 months postpartum.

\section{Nursing analyses}

Significant differences in memory were observed among those fed directly at the breast vs those fed partially/completely by bottle. Specifically, for relational memory at 6 months, in the lag 2 trials, which encompassed both delay and interfering information, the proportion of time spent looking at the correctly matched picture in the third 1000-ms time bin was higher among those who received milk directly from the breast than among those fed partially/completely by bottle $(P=0.022)$ (Table $3 \mathrm{a})$. No significant differences were observed by the type of nursing in the lag 0 trials, which involved neither delay nor interference from other stimuli (Table 3a). At 41 months, children fed directly at the breast were accurate in a higher proportion of trials than were those fed breast milk partially/completely by bottle in an aspect of the relational memory task that included face stimuli $(P=0.038)$. Children fed at the breast only also spent proportionally longer time looking at the correctly matched picture in the lag 2 trials conducted at 54 months $(P=0.031)$ (Table 3a).

During the deferred imitation test, the number of target behaviors reproduced by 6 -month-old infants was greater among those who were fed directly at the breast than among those bottle-fed breast milk $(P=0.043)$. Performance in other memory tasks, including habituation and deferred imitation at time points other than 6 months, was similar across the different types of nursing (Supplementary Table 2a, Online Resource 1). 
Table 3 Associations between (a) the type of nursing and (b) milk nutrients with performance in relational binding

\begin{tabular}{|c|c|c|c|c|c|c|c|c|}
\hline \multirow{3}{*}{$\begin{array}{l}\text { Relational binding } \\
\text { (memory) }\end{array}$} & \multirow{3}{*}{$\mathrm{N}$} & \multicolumn{3}{|l|}{ (a) Nursing } & \multirow{3}{*}{$\mathrm{N}$} & \multicolumn{3}{|l|}{ (b) Nutrients } \\
\hline & & \multicolumn{2}{|c|}{ Unadjusted mean $\pm \mathrm{SD}$} & \multirow{2}{*}{$\begin{array}{l}\text { Adjusted mean } \\
\text { differences }(95 \% \\
\text { CI })^{\text {b, d }} \\
\text { At breast only }\end{array}$} & & \multicolumn{2}{|c|}{ Unadjusted mean $\pm \mathrm{SD}$} & \multirow{2}{*}{$\begin{array}{l}\text { Adjusted mean dif- } \\
\text { ferences }(95 \% \mathrm{CI})^{\mathrm{c}, \mathrm{d}} \\
\text { Breast milk + for- } \\
\text { mula or breast milk } \\
\text { only }\end{array}$} \\
\hline & & $\begin{array}{l}\text { Breast }+ \text { bottle } \\
\text { or bottle only }\end{array}$ & At breast only & & & Formula only & $\begin{array}{l}\text { Breast milk + for- } \\
\text { mula or breast milk } \\
\text { only }\end{array}$ & \\
\hline \multicolumn{9}{|l|}{6 months } \\
\hline \multicolumn{9}{|c|}{ Lag 0 trials (Time bins ${ }^{\mathrm{a}}$ ) } \\
\hline 1000-ms Bin 1 & 34 & $0.31 \pm 0.17$ & $0.41 \pm 0.21$ & $0.13(-0.07,0.33)$ & 90 & $0.36 \pm 0.22$ & $0.23 \pm 0.14$ & $-0.10(-0.23,0.04)$ \\
\hline 1000-ms Bin 2 & 31 & $0.28 \pm 0.19$ & $0.32 \pm 0.21$ & $-0.04(-0.20,0.11)$ & 85 & $0.32 \pm 0.26$ & $0.28 \pm 0.21$ & $-0.02(-0.18,0.13)$ \\
\hline 1000-ms Bin 3 & 29 & $0.30 \pm 0.36$ & $0.27 \pm 0.28$ & $-0.08(-0.46,0.31)$ & 78 & $0.30 \pm 0.32$ & $0.24 \pm 0.23$ & $-0.14(-0.35,0.06)$ \\
\hline \multicolumn{9}{|c|}{ Lag 2 trials $\left(\right.$ Time bins $\left.{ }^{\mathrm{a}}\right)$} \\
\hline 1000-ms Bin 1 & 33 & $0.43 \pm 0.29$ & $0.42 \pm 0.31$ & $-0.07(-0.36,0.21)$ & 85 & $0.36 \pm 0.23$ & $0.43 \pm 0.17$ & $0.07(-0.07,0.22)$ \\
\hline 1000-ms Bin 2 & 28 & $0.42 \pm 0.17$ & $0.40 \pm 0.32$ & $0.08(-0.19,0.35)$ & 81 & $0.35 \pm 0.24$ & $0.36 \pm 0.29$ & $0.06(-0.11,0.24)$ \\
\hline 1000-ms Bin 3 & 24 & $0.29 \pm 0.26$ & $0.43 \pm 0.30$ & $0.41(0.07,0.74)^{\mathrm{e}}$ & 70 & $0.46 \pm 0.34$ & $0.40 \pm 0.33$ & $0.04(-0.21,0.29)$ \\
\hline \multicolumn{9}{|l|}{41 months } \\
\hline $\begin{array}{l}\text { Accuracy in food } \\
\text { block }\end{array}$ & 57 & $3.13 \pm 1.07$ & $2.84 \pm 0.85$ & $-0.27(-0.86,0.32)$ & 109 & $2.89 \pm 1.18$ & $3.00 \pm 1.06$ & $0.25(-0.41,0.91)$ \\
\hline $\begin{array}{l}\text { Accuracy in face } \\
\text { block }\end{array}$ & 57 & $2.35 \pm 0.88$ & $2.92 \pm 1.09$ & $0.67(0.04,1.29)^{\mathrm{e}}$ & 108 & $2.56 \pm 1.09$ & $2.63 \pm 0.77$ & $0.30(-0.29,0.89)$ \\
\hline $\begin{array}{l}\text { Combined food } \\
\text { and face accu- } \\
\text { racy }\end{array}$ & 58 & $5.41 \pm 1.52$ & $5.65 \pm 1.44$ & $0.45(-0.49,1.39)$ & 109 & $5.42 \pm 1.67$ & $5.63 \pm 1.41$ & $0.59(-0.33,1.51)$ \\
\hline $\begin{array}{l}\text { Inference memory } \\
\text { accuracy }\end{array}$ & 56 & $1.23 \pm 0.76$ & $1.40 \pm 0.91$ & $0.29(-0.25,0.84)$ & 106 & $1.61 \pm 0.87$ & $1.25 \pm 0.68$ & $-0.15(-0.64,0.34)$ \\
\hline \multicolumn{9}{|l|}{54 months } \\
\hline \multicolumn{9}{|l|}{ Lag 0 trials } \\
\hline Accuracy & 52 & $0.55 \pm 0.29$ & $0.67 \pm 0.31$ & $-0.03(-0.26,0.20)$ & 115 & $0.55 \pm 0.31$ & $0.59 \pm 0.26$ & $0.02(-0.14,0.19)$ \\
\hline $\begin{array}{l}\% \text { Looking to } \\
\text { correct match }\end{array}$ & 52 & $0.37 \pm 0.17$ & $0.40 \pm 0.16$ & $-0.09(-0.23,0.04)$ & 114 & $0.43 \pm 0.21$ & $0.45 \pm 0.17$ & $0.01(-0.11,0.11)$ \\
\hline \multicolumn{9}{|l|}{ Lag 2 trials } \\
\hline Accuracy & 52 & $0.41 \pm 0.22$ & $0.49 \pm 0.32$ & $0.08(-0.15,0.32)$ & 115 & $0.41 \pm 0.27$ & $0.53 \pm 0.26$ & $0.13(-0.02,0.28)$ \\
\hline $\begin{array}{l}\text { \% Looking to } \\
\text { correct match }\end{array}$ & 52 & $0.36 \pm 0.15$ & $0.39 \pm 0.09$ & $0.12(0.01,0.22)^{\mathrm{e}}$ & 113 & $0.34 \pm 0.13$ & $0.42 \pm 0.12$ & $0.06(-0.01,0.13)$ \\
\hline
\end{tabular}

${ }^{a}$ Time bins are defined in 1000-ms blocks after the pictures appear on the screen

${ }^{\mathrm{b}}$ Values are adjusted mean differences $(95 \% \mathrm{CI}$ ) from the reference group (Breast + bottle or bottle only)

${ }^{\mathrm{c}}$ Values are adjusted mean differences (95\% CI) from the reference group (Formula only)

${ }^{\mathrm{d}}$ Values are adjusted for ethnicity (Chinese, Malay and Indian), maternal education (non-tertiary and tertiary), birth weight category (SGA, AGA, and LGA), 26-week STAI-state scores (continuous), child's sex, and age during assessment (continuous)

${ }^{\mathrm{e}}$ Values are $P<0.05$ compared to the reference group

Performance on testing batteries conducted at 24,48 and 54 months are shown in Table 4 and Supplementary Table 3 (Online Resource 1). Among children "nursed" differently, a significant difference was observed for The Peabody Picture Vocabulary Test (PPVT) and for Weber Fraction, a part of Panamath; contrary to our hypothesis, children fed directly at the breast performed less well than those fed partially/completely by bottle, $P=0.039$ and $P=0.013$, respectively (Supplementary Table 3a, Online Resource 1). No other significant associations were observed. No significant associations were observed between type of nursing and tasks relating to executive functioning (dimensional card sorting tasks, sticker and snack delay), attention (visual expectation and CANTAB) or social-emotional development (novel word learning) (Supplementary Table 4a-6a, Online Resource 1). 
Table 4 Associations between (a) the type of nursing and (b) milk nutrients with testing batteries

\begin{tabular}{|c|c|c|c|c|c|c|c|c|}
\hline \multirow[t]{3}{*}{ Testing batteries } & \multicolumn{4}{|c|}{ (a) Nursing } & \multicolumn{4}{|c|}{ (b) Nutrients } \\
\hline & \multirow[t]{2}{*}{$N$} & \multicolumn{2}{|c|}{ Unadjusted mean $\pm \mathrm{SD}$} & \multirow{2}{*}{$\begin{array}{l}\text { Adjusted mean } \\
\text { differences }(95 \% \\
\mathrm{CI})^{\mathrm{a}, \mathrm{c}} \\
\text { At breast only }\end{array}$} & \multirow[t]{2}{*}{$N$} & \multicolumn{2}{|c|}{ Unadjusted mean $\pm \mathrm{SD}$} & \multirow{2}{*}{$\begin{array}{l}\text { Adjusted mean } \\
\text { differences }(95 \% \\
\text { CI })^{\text {b,c }} \\
\text { Breast milk }+ \text { for- } \\
\text { mula or breast milk } \\
\text { only }\end{array}$} \\
\hline & & $\begin{array}{l}\text { Breast + bottle } \\
\text { or bottle only }\end{array}$ & At breast only & & & Formula only & $\begin{array}{l}\text { Breast milk }+ \text { for- } \\
\text { mula or breast milk } \\
\text { only }\end{array}$ & \\
\hline \multicolumn{9}{|l|}{ BSID-III } \\
\hline \multicolumn{9}{|l|}{24 months } \\
\hline Cognition & 61 & $11.45 \pm 2.56$ & $10.29 \pm 2.48$ & $-1.11(-2.55,0.33)$ & ) 157 & $9.62 \pm 2.43$ & $11.06 \pm 2.24$ & $1.36(0.32,2.40)^{\mathrm{d}}$ \\
\hline $\begin{array}{l}\text { Receptive lan- } \\
\text { guage }\end{array}$ & 61 & $10.15 \pm 2.84$ & $9.86 \pm 2.03$ & $-0.21(-1.76,1.33)$ & 156 & $8.25 \pm 2.58$ & $9.65 \pm 3.16$ & $0.48(-0.70,1.65)$ \\
\hline $\begin{array}{l}\text { Expressive lan- } \\
\text { guage }\end{array}$ & 61 & $9.97 \pm 2.49$ & $10.29 \pm 2.77$ & $-0.06(-1.57,1.46)$ & 155 & $8.44 \pm 2.15$ & $9.58 \pm 3.10$ & $0.57(-0.47,1.60)$ \\
\hline Fine motor & 61 & $10.45 \pm 1.87$ & $11.25 \pm 2.27$ & $1.08(-0.15,2.31)$ & 154 & $10.35 \pm 2.27$ & $11.17 \pm 2.57$ & $0.64(-0.56,1.83)$ \\
\hline Gross motor & 61 & $11.91 \pm 3.53$ & $11.82 \pm 2.75$ & $-0.33(-2.30,1.64)$ & ) 154 & $10.71 \pm 3.00$ & $12.17 \pm 3.12$ & $1.60(0.09,3.10)^{\mathrm{d}}$ \\
\hline \multicolumn{9}{|l|}{ KBIT-2 } \\
\hline \multicolumn{9}{|l|}{54 months } \\
\hline Verbal & 62 & $93.85 \pm 13.32$ & $95.62 \pm 19.42$ & $1.47(-9.53,12.46)$ & ) 158 & $79.87 \pm 13.06$ & $91.16 \pm 17.15$ & $6.50(0.13,12.87)^{\mathrm{d}}$ \\
\hline Nonverbal & 62 & $100.52 \pm 15.99$ & $106.17 \pm 10.30$ & $4.72(-4.30,13.75)$ & 159 & $95.71 \pm 15.16$ & $102.28 \pm 13.32$ & $6.28(-0.56,13.11)$ \\
\hline IQ & 62 & $96.91 \pm 13.95$ & $101.34 \pm 13.19$ & $3.76(-5.75,13.27)$ & 158 & $86.10 \pm 13.56$ & $96.81 \pm 14.03$ & $7.59(1.20,13.99)^{\mathrm{d}}$ \\
\hline
\end{tabular}

BSID-III Bayley Scales of Infant and Toddler Development (Third Edition), KBIT-2 Kaufman Brief Intelligence Test (Second Edition)

${ }^{a}$ Values are adjusted mean differences $(95 \% \mathrm{CI})$ from the reference group (Breast + bottle or bottle only)

${ }^{\mathrm{b}}$ Values are adjusted mean differences (95\% CI) from the reference group (Formula only)

${ }^{\mathrm{c}}$ Adjusted models include the covariates: ethnicity (Chinese, Malay, and Indian), maternal education (non-tertiary and tertiary), birth weight category (SGA, AGA, and LGA), 26-week STAI-state scores (continuous) and child's sex

${ }^{\mathrm{d}}$ Values are $P<0.05$ compared to the reference group

\section{Nutrient analyses}

Among all children who were bottle-fed during infancy, type of milk (breast milk vs formula) consumed was not significantly associated with performance in the memory tasks conducted at any follow-up time point (Table 4b, and Supplementary Table 2b, Online Resource 1).

Results showed an overall positive crude association between breast milk feeding and cognition domain scores, as well as gross motor scores, on the BSID-III (Table 4b). Even after adjusting for confounders, children who were fed some/only breast milk in the first 3 months had significantly higher cognition domain scores $(P=0.011)$, as well as gross motor scores than those who were fed only formula $(P=0.038)$. Children who were fed some/only breast milk also scored higher for the verbal component of the KBIT at 54 months than those who were fed formula only $(P=0.046)$; the overall score on the KBIT was also significantly higher $(P=0.020)$. No significant associations were observed between milk types and any of the school readiness tests at 48 months (Supplementary Table 3b, Online Resource 1).
Children who had been fed some/all breast milk had better use of strategy in the spatial working memory task than those fed formula only $(P=0.023)$ (Supplementary Table 5b, Online Resource 1). No significant associations were observed on tasks of executive functioning (dimensional card sorting, sticker and snack delay), attention (visual expectation) or social-emotional development (novel word learning) (Supplementary Tables 4-6, Online Resource 1). F-statistic and $P$ values for the associations of the type of nursing or milk nutrients with cognitive assessments are shown in Supplementary Tables 7-11, Online Resource 1.

\section{Discussion}

Our results suggest that contact accompanying feeding directly at the breast may contribute to brain development. This is consistent with prior, unexamined, hypotheses that the physical and emotional contact of direct breastfeeding (the nursing), in addition to the nutritional content of breast milk may confer benefits in child cognition. Here, we observed that whilst breast milk can improve the child's 
general cognition, motor skills, as well as language abilities, direct breastfeeding appears to influence their memory.

Compared to children fed infant formula only during early infancy, those fed expressed breast milk demonstrated significantly better cognitive performance at 2 and 4.5 years, even after adjusting for maternal education, age and anxiety level during pregnancy. Higher IQ scores at 4.5 years appear to be driven by improved verbal skills; the association between breast milk intake and higher scores on nonverbal tasks was of only borderline statistical significance. We observed no significant differences in 2-year-old language tasks, nor on any of the 4-year-old school readiness tests, although the mean scores for those who consumed breast milk were generally higher. Results of previous breastfeeding and cognition studies are not directly comparable to ours, because in past work "breastfeeding" refers to infants fed directly at the breast and/or fed expressed breast milk. Nevertheless, many studies have reported better cognitive performance [3, 29, $30]$ and language abilities [3, 5, 30] among children who had consumed more breast milk as infants. Various milk nutrients have been hypothesized to contribute to improved child cognitive ability, including long-chain polyunsaturated fatty acids, such as AA and DHA [31-33] (which are important for cognitive maturation [34]). Nonetheless, randomized trials of feeding formula supplemented with these nutrients have not confirmed those hypotheses [35].

GUSTO children fed expressed breast milk also demonstrated better gross motor skills at age 2 years than those fed formula only. Previous studies of motor skills in relation to breastfeeding have reported inconsistent results $[1,12$, $30,36,37]$. Even among studies that conducted the same motor tests (i.e., BSID) at approximately 2 years of age have reported mixed results $[30,36]$. One explanation for this disparity is that past work did not examine both nutritional and nursing influences on motor development. Further studies with larger samples are needed.

Among GUSTO children who were exclusively fed breast milk, those fed directly at the breast scored higher on several memory tasks compared to children fed breast milk via bottles. In particular, they reproduced more target actions during the deferred imitation task at 6 months and showed evidence of better relational binding at 6,41 and 54 months of age. Deferred imitation requires a child to reproduce previously learned actions and so indicates recollection of past events. The relational binding task requires children to bind together different aspects of an experience, scene, etc., and is important to autobiographical memory and learning [17]. Both deferred imitation [38] and relational binding [39, 40] may reflect memory processes that primarily involve the hippocampus, a region of the brain essential for flexible memory expression [41].

How the act of breastfeeding benefits memory is unknown. The benefits are unlikely due to differences in the feeding frequencies, as the nutrients that contribute to infant satiety, and, therefore, to feeding frequency, are nearly identical for both modes of breast milk feeding. The benefits to memory may be due to differences in the frequency and/ or duration of mother-infant contact. For example, direct skin-to-skin contact, perhaps more likely in children fed at the breast, may influence a variety of processes including pain sensitivity and stress responsivity. Variation in stress may be especially influential to memory processes. Many studies have reported that exposure to stress or an elevated level of corticosteroids alters performance on memory tasks that are dependent on the hippocampus $[42,43]$. In animal studies, stress alters ensuing synaptic plasticity and firing properties of hippocampal neurons. Additionally, both human and animal studies have shown that stress can change neuronal morphology, suppress neuronal proliferation, alter hippocampal volume [44, 45], and, perhaps alter the time course of hippocampal growth [46]. Varying levels of hypothalamic-pituitary-adrenal axis neuroendocrine hormones, particularly glucocorticoids, appear to mediate the myriad stress effects on the hippocampus [45].

Our study's strengths include assessment of numerous specific cognitive measures, as well as the use of generalized cognitive test batteries. Moreover, cognitive measures were obtained at several time points from early infancy to 4.5 years. We were also able to control for a large number of potential confounding factors. One study limitation is our definition of the type of nursing, which was defined at 3 months of age. As a result, we were unable to examine whether the neurocognitive outcomes would be similar if the type of nursing was also compared at later ages. However, of the mothers who continued to breastfeed to 6 months $(<50 \%$ of the cohort), the majority ( $>70 \%)$ maintained the same type of nursing at 3 and 6 months, suggesting that nursing type at 3 months is a valid surrogate of longer term feeding. We also have modest statistical power for some analyses, owing to small sample sizes for some cognitive measures conducted. Finally, we examined many cognitive outcomes, most of the associations we observed were of modest magnitude, and some were opposite in direction to our hypothesis. Some of our results may, therefore, reflect the play of chance.

Nevertheless, ours is the first study that has attempted to disentangle the potential effects on child cognitive ability of the nutrients in breast milk vs the act of nursing implicit in direct breastfeeding.

Our results suggest that breastfeeding's impact on brain development may be due to both factors. Although the significant associations we observed were modest in magnitude and limited to some tests at specific ages, our findings suggest that the nutritional content of breast milk may improve general child cognition, language abilities and gross motor skills, while feeding infants directly at the breast may 
influence memory abilities. Such work may be of direct relevance to maternal child postpartum well-being and pediatric practice: anecdotally, mothers often interpret advice to breastfeed as advice to provide breast milk, and pumping breast milk may be a preferred means of administration in some cultures. As breast-pump technology becomes increasingly advanced and accessible, providing breast milk may become further removed from at-the-breast feeding. Future studies with larger sample sizes and higher exclusive breastfeeding rates will be important to confirm or refute our findings.

Acknowledgements We wish to thank participants of the GUSTO study, all staff involved in the collection and management of data, and the GUSTO study group. The GUSTO study group includes Allan Sheppard, Amutha Chinnadurai, Anne Eng Neo Goh, Anne RifkinGraboi, Anqi Qiu, Arijit Biswas, Bee Wah Lee, Birit F.P. Broekman, Boon Long Quah, Borys Shuter, Chai Kiat Chng, Cheryl Ngo, Choon Looi Bong, Christiani Jeyakumar Henry, Claudia Chi, Cornelia Yin Ing Chee, Yam Thiam Daniel Goh, Doris Fok, E Shyong Tai, Elaine Tham, Elaine Quah Phaik Ling, Evelyn Chung Ning Law, Evelyn Xiu Ling Loo, Fabian Yap, Falk Mueller-Riemenschneider, George Seow Heong Yeo, Helen Chen, Heng Hao Tan, Hugo P S van Bever, Iliana Magiati, Inez Bik Yun Wong, Ivy Yee-Man Lau, Izzuddin Bin Mohd Aris, Jeevesh Kapur, Jenny L. Richmond, Jerry Kok Yen Chan, Joanna D. Holbrook, Joanne Yoong, Joao N. Ferreira., Jonathan Tze Liang Choo, Jonathan Y. Bernard, Joshua J. Gooley, Keith M. Godfrey, Kenneth Kwek, Kok Hian Tan, Krishnamoorthy Niduvaje, Kuan Jin Lee, Leher Singh, Lieng Hsi Ling, Lin Lin Su, Ling-Wei Chen, Lourdes Mary Daniel, Lynette P Shek, Marielle V. Fortier, Mark Hanson, Mary Foong-Fong Chong, Mary Rauff, Mei Chien Chua, Melvin Khee-Shing Leow, Michael Meaney, Mya Thway Tint, Neerja Karnani, Ngee Lek, Oon Hoe Teoh, P. C. Wong, Paulin Tay Straughan, Peter D. Gluckman, Pratibha Agarwal, Queenie Ling Jun Li, Rob M. van Dam, Salome A. Rebello, Seang-Mei Saw, See Ling Loy, S. Sendhil Velan, Seng Bin Ang, Shang Chee Chong, Sharon Ng, Shiao-Yng Chan, Shirong Cai, Shu-E Soh, Sok Bee Lim, Stella Tsotsi, Chin-Ying Stephen Hsu, Sue Anne Toh, Swee Chye Quek, Victor Samuel Rajadurai, Walter Stunkel, Wayne Cutfield, Wee Meng Han, Wei Wei Pang, Yap-Seng Chong, Yin Bun Cheung, Yiong Huak Chan and Yung Seng Lee.

Funding This research was supported by the Singapore National Research Foundation under its Translational and Clinical Research (TCR) Flagship Programme and administered by the Singapore Ministry of Health's National Medical Research Council (NMRC), Singapore-NMRC/TCR/004-NUS/2008; NMRC/TCR/012-NUHS/2014. KMG is supported by the UK Medical Research Council (MC UU_12011/4), the National Institute for Health Research (as an NIHR Senior Investigator (NF-SI-0515-10042) and through the NIHR Southampton Biomedical Research Centre) and the European Union's Erasmus + Capacity-Building ENeA SEA Project and Seventh Framework Programme (FP7/2007-2013), projects EarlyNutrition and ODIN under grant agreement numbers 289346 and 613977 . Additional funding was provided by the Singapore Institute for Clinical Sciences, Agency for Science Technology and Research (A*STAR), and Abbott Nutrition Research and Development Asia-Pacific Center.

\section{Compliance with ethical standards}

Conflict of interest KMG, LPS and Y-SC has received reimbursement for speaking at conferences sponsored by companies selling nutritional products. KMG, S-YC and Y-SC are part of an academic consortium that has received research funding from Abbott Nutrition, Nestec and Danone. The other authors have no financial or personal conflict of interest to declare.

Open Access This article is distributed under the terms of the Creative Commons Attribution 4.0 International License (http://creativeco mmons.org/licenses/by/4.0/), which permits unrestricted use, distribution, and reproduction in any medium, provided you give appropriate credit to the original author(s) and the source, provide a link to the Creative Commons license, and indicate if changes were made.

\section{References}

1. Angelsen NK, Vik T, Jacobsen G, Bakketeig LS (2001) Breast feeding and cognitive development at age 1 and 5 years. Arch Dis Child 85:183-188

2. Daniels MC, Adair LS (2005) Breast-feeding influences cognitive development in Filipino children. J Nutr 135:2589-2595

3. Kramer MS, Aboud F, Mironova E et al (2008) Breastfeeding and child cognitive development: new evidence from a large randomized trial. Arch Gen Psychiatry 65:578-584. https://doi. org/10.1001/archpsyc.65.5.578

4. Cai S, Pang WW, Low YL et al (2015) Infant feeding effects on early neurocognitive development in Asian children. Am J Clin Nutr 101:326-336. https://doi.org/10.3945/ajcn.114.095414

5. Bernard JY, De Agostini M, Forhan A et al (2013) Breastfeeding duration and cognitive development at 2 and 3 years of age in the EDEN mother-child cohort. J Pediatr 163:36-42 e31. https://doi. org/10.1016/j.jpeds.2012.11.090

6. Horta BL, Loret de Mola C, Victora CG (2015) Breastfeeding and intelligence: a systematic review and meta-analysis. Acta Paediatr 104:14-19. https://doi.org/10.1111/apa.13139

7. Innis SM (1991) Essential fatty acids in growth and development. Prog Lipid Res 30:39-103

8. Innis SM (2003) Perinatal biochemistry and physiology of longchain polyunsaturated fatty acids. J Pediatr 143:S1-S8

9. Tram TH, Brand Miller JC, McNeil Y, McVeagh P (1997) Sialic acid content of infant saliva: comparison of breast fed with formula fed infants. Arch Dis Child 77:315-318

10. Wang B, Miller JB, Sun Y et al (2001) A longitudinal study of salivary sialic acid in preterm infants: comparison of human milkfed versus formula-fed infants. J Pediatr 138:914-916. https://doi. org/10.1067/mpd.2001.113044

11. Bourre JM (2006) Effects of nutrients (in food) on the structure and function of the nervous system: update on dietary requirements for brain. Part 1: micronutrients. J Nutr Health Aging 10:377-385

12. Deoni SC, Dean DC III, Piryatinsky I et al (2013) Breastfeeding and early white matter development: A cross-sectional study. Neuroimage 82:77-86. https://doi.org/10.1016/j.neuroimage .2013 .05 .090

13. Newton N (1971) The uniqueness of human milk. Psychological differences between breast and bottle feeding. Am J Clin Nutr 24:993-1004

14. Stuart-Macadam P, Dettwyler KA (1995) Breastfeeding: biocultural perspectives. Aldine de Gruyter, New York

15. Kim P, Feldman R, Mayes LC et al (2011) Breastfeeding, brain activation to own infant cry, and maternal sensitivity. $\mathrm{J}$ Child Psychol Psychiatry 52:907-915. https://doi.org/10.111 1/j.1469-7610.2011.02406.x

16. Baumwell L, Tamis-LeMonda CS, Bornstein MH (1997) Maternal verbal sensitivity and child language comprehension. 
Infant Behav Dev 20:247-258. https://doi.org/10.1016/S0163 $-6383 \% 2897 \% 2990026-6$

17. Cleveland L, Hill CM, Pulse WS et al (2017) Systematic review of skin-to-skin care for full-term, healthy newborns. J Obstet Gynecol Neonatal Nurs 46:857-869. https://doi.org/10.1016/j. jogn.2017.08.005

18. Binns CW, Win NN, Zhao Y, Scott JA (2006) Trends in the expression of breastmilk 1993-2003. Breastfeed Rev 14:5-9

19. Hornbeak DM, Dirani M, Sham WK et al (2010) Emerging trends in breastfeeding practices in Singaporean Chinese women: findings from a population-based study. Ann Acad Med Singap 39:88-94

20. Bai DL, Fong DY, Lok KY et al (2017) Practices, predictors and consequences of expressed breast-milk feeding in healthy full-term infants. Public Health Nutr 20:492-503. https://doi. org/10.1017/S136898001600241X

21. Lucas A, Morley R, Cole TJ et al (1992) Breast milk and subsequent intelligence quotient in children born preterm. Lancet 339:261-264

22. Pang WW, Aris IM, Fok D et al (2016) Determinants of breastfeeding practices and success in a multi-ethnic asian population. Birth 43:68-77. https://doi.org/10.1111/birt.12206

23. Soh SE, Tint MT, Gluckman PD et al (2014) Cohort profile: growing up in singapore towards healthy outcomes (GUSTO) birth cohort study. Int J Epidemiol 43:1401-1409. https://doi. org/10.1093/ije/dyt125

24. Mikolajczyk RT, Zhang J, Betran AP et al (2011) A global reference for fetal-weight and birthweight percentiles. Lancet 377:1855-1861. https://doi.org/10.1016/S0140-6736(11)60364-4

25. Qiu A, Rifkin-Graboi A, Chen H et al (2013) Maternal anxiety and infants' hippocampal development: timing matters. Transl Psychiatry 3:e306. https://doi.org/10.1038/tp.2013.79

26. Pang WW, Bernard JY, Thavamani G et al (2017) Direct vs. expressed breast milk feeding: relation to duration of breastfeeding. Nutrients. https://doi.org/10.3390/nu9060547

27. Labiner-Wolfe J, Fein SB, Shealy KR, Wang C (2008) Prevalence of breast milk expression and associated factors. Pediatrics 122(Suppl 2):S63-S68. https://doi.org/10.1542/peds.2008-1315h

28. Geraghty S, Davidson B, Tabangin M, Morrow A (2012) Predictors of breastmilk expression by 1 month postpartum and influence on breastmilk feeding duration. Breastfeed Med 7:112-117. https ://doi.org/10.1089/bfm.2011.0029

29. Brion MJ, Lawlor DA, Matijasevich A et al (2011) What are the causal effects of breastfeeding on IQ, obesity and blood pressure? Evidence from comparing high-income with middle-income cohorts. Int J Epidemiol 40:670-680. https://doi.org/10.1093/ije/ dyr020

30. Leventakou V, Roumeliotaki T, Koutra K et al (2015) Breastfeeding duration and cognitive, language and motor development at 18 months of age: Rhea mother-child cohort in Crete, Greece. J Epidemiol Community Health 69:232-239. https://doi.org/10.1136/ jech-2013-202500
31. Koletzko B, Agostoni C, Carlson SE et al (2001) Long chain polyunsaturated fatty acids (LC-PUFA) and perinatal development. Acta Paediatr 90:460-464

32. Farquharson J, Cockburn F, Patrick WA et al (1992) Infant cerebral cortex phospholipid fatty-acid composition and diet. Lancet 340:810-813

33. Isaacs EB, Fischl BR, Quinn BT et al (2010) Impact of breast milk on intelligence quotient, brain size, and white matter development. Pediatr Res 67:357-362. https://doi.org/10.1203/PDR.0b013 e3181d026da

34. O'Connor DL, Hall R, Adamkin D et al (2001) Growth and development in preterm infants fed long-chain polyunsaturated fatty acids: a prospective, randomized controlled trial. Pediatrics 108:359-371

35. Jasani B, Simmer K, Patole SK, Rao SC (2017) Long chain polyunsaturated fatty acid supplementation in infants born at term. Cochrane Database Syst Rev 3:CD000376. https://doi. org/10.1002/14651858.CD000376.pub4

36. Rogan WJ, Gladen BC (1993) Breast-feeding and cognitive development. Early Hum Dev 31:181-193

37. Vestergaard M, Obel C, Henriksen TB et al (1999) Duration of breastfeeding and developmental milestones during the latter half of infancy. Acta Paediatr 88:1327-1332

38. McDonough L, Mandler JM, McKee RD, Squire LR (1995) The deferred imitation task as a nonverbal measure of declarative memory. Proc Natl Acad Sci USA 92:7580-7584

39. Shimamura AP (2010) Hierarchical relational binding in the medial temporal lobe: the strong get stronger. Hippocampus 20:1206-1216. https://doi.org/10.1002/hipo.20856

40. Hannula DE, Ranganath C (2008) Medial temporal lobe activity predicts successful relational memory binding. J Neurosci 28:116-124. https://doi.org/10.1523/JNEUROSCI.3086-07.2008

41. Richmond J, Nelson CA (2009) Relational memory during infancy: evidence from eye tracking. Dev Sci 12:549-556. https ://doi.org/10.1111/j.1467-7687.2009.00795.x

42. McEwen BS, Sapolsky RM (1995) Stress and cognitive function. Curr Opin Neurobiol 5:205-216

43. Kim JJ, Diamond DM (2002) The stressed hippocampus, synaptic plasticity and lost memories. Nat Rev Neurosci 3:453-462. https ://doi.org/10.1038/nrn849

44. Cameron HA, Schoenfeld TJ (2018) Behavioral and structural adaptations to stress. Front Neuroendocrinol 49:106-113. https ://doi.org/10.1016/j.yfrne.2018.02.002

45. Kim EJ, Pellman B, Kim JJ (2015) Stress effects on the hippocampus: a critical review. Learn Mem 22:411-416. https://doi. org/10.1101/lm.037291.114

46. Rifkin-Graboi A, Kong L, Sim LW et al (2015) Maternal sensitivity, infant limbic structure volume and functional connectivity: a preliminary study. Transl Psychiatry 5:e668. https://doi. org/10.1038/tp.2015.133

\section{Affiliations}

\section{Wei Wei Pang ${ }^{1}$ - Pei Ting Tan ${ }^{1}$. Shirong Cai ${ }^{1,2}$ - Doris Fok ${ }^{1}$ - Mei Chien Chua ${ }^{3}$. Sock Bee Lim ${ }^{4} \cdot$ Lynette P. Shek $^{2,5,6}$. Shiao-Yng Chan $^{1,2} \cdot$ Kok Hian Tan $^{7,8} \cdot$ Fabian Yap ${ }^{9}$. Peter D. Gluckman ${ }^{2,10} \cdot$ Keith M. Godfrey $^{11,12}$. Michael J. Meaney ${ }^{2,13,14} \cdot$ Birit F. P. Broekman ${ }^{15}$. Michael S. Kramer ${ }^{1,16,17} \cdot$ Yap-Seng Chong ${ }^{1,2} \cdot$ Anne Rifkin-Graboi ${ }^{2,18}$}

1 Department of Obstetrics and Gynaecology, Yong Loo Lin School of Medicine, National University of Singapore, National University Health System, Singapore 119228, Singapore
2 Singapore Institute for Clinical Sciences (SICS), Agency for Science, Technology and Research (A*STAR), Singapore 117609, Singapore 
3 Department of Neonatology, KK Women's and Children's Hospital, Singapore 229899, Singapore

4 Department of Child Development, KK Women's and Children's Hospital, Singapore 229899, Singapore

5 Department of Paediatrics, Yong Loo Lin School of Medicine, National University of Singapore, National University Health System, Singapore 119228, Singapore

6 Khoo Teck Puat-National University Children's Medical Institute, National University Health System, Singapore 119228, Singapore

7 Department of Maternal Fetal Medicine, KK Women's and Children's Hospital, Singapore 229899, Singapore

8 Duke-NUS Medical School, Singapore 169857, Singapore

9 Department of Pediatric Endocrinology, KK Women's and Children's Hospital, Singapore 229899, Singapore

10 Liggins Institute, University of Auckland, 1142 Auckland, New Zealand

11 Medical Research Council Lifecourse Epidemiology Unit, SO16 6YD Southampton, UK
12 NIHR Southampton Biomedical Research Centre, University of Southampton and University Hospital Southampton NHS Foundation Trust, SO16 6YD Southampton, UK

13 Departments of Psychiatry and Neurology, McGill University, Montreal, QC, Canada

14 Ludmer Centre for Neuroinformatics and Mental Health, Montreal, QC, Canada

15 Department of Psychiatry, VU Medical Centre, Amsterdam, The Netherlands

16 Department of Pediatrics, Faculty of Medicine, McGill University, Montreal, QC H3A 1A2, Canada

17 Department of Epidemiology, Biostatistics and Occupational Health, Faculty of Medicine, McGill University, Montreal, QC H3A 1A2, Canada

18 National Institute of Education, 1 Nanyang Walk, Singapore 637616, Singapore 